\title{
Avaliação de RPAs para pulverização em diferentes culturas
}

\author{
Evaluation of RPAs for spraying in differents cultures
}

Joissy Mayara de Almeida Andrade
Universidade Estadual Paulista (UNESP) e Eficiente Soluções Florestais Ltda
(Autor para correspondência).
joissy.andrade@gmail.com
Dennis R. Pretto
Eficiente Soluções Florestais Ltda, $+55163617-2274$, Brasil.
denispretto@eficientesf.com.br
Eglairto V. Carvalho
Eficiente Soluções Florestais Ltda, $+55163617-2274$, Brasil.
eglairtocarvalho@eficientesf.com.b
Denizart Bolonhezi
Agência Paulista de Tecnologia dos Agronegócio, APTA-SAA, $+55163919-9920$, Brasil.
denizart@apta.sp.gov.b
Jose Roberto Scarpellini
Agência Paulista de Tecnologia dos Agronegócio, APTA-SAA, $+55163919-9920$, Brasil.
jrscarpellini@apta.sp.gov.br
Bruna Cardoso Vieira
Agência Paulista de Tecnologia dos Agronegócio, APTA-SAA, $+55163919-9920$, Brasil.
brunamvcar@hotmail.com

\section{Resumo}

O presente trabalho teve como objetivo estudar e validar a aplicação de defensivos através de RPAs (Aeronave Remotamente Pilotadas), comumente denominadas de Drones. O experimento foi realizado na APTA Centro Leste no município do Ribeirão Preto, SP. Foi utilizado a: solução de herbicida glifosate $1.5 \mathrm{~kg} \mathrm{ha}^{-1}$ (Roundup WG ${ }^{\circledR}$ ) utilizando $15 \mathrm{~L} \mathrm{ha}^{-1}$ de volume de calda. O RPA que sobrevoou a área de estudo em três diferentes altitudes, juntamente com três diferentes modelos de bicos pulverizadores em uma faixa compreendida de $50 \mathrm{~m}$ x $5 \mathrm{~m}$, sendo que nessas faixas foram distribuídos papéis sensíveis à água, para verificar o tamanho, quantidade e distribuição de gotas que caíram sobre as plantas. Uma vez contabilizadas as informações, esperou-se que fosse possível criar diferentes modelos de aplicações, levando a uma maior eficiência e eficácia das aplicações. Além disso, evitar desperdício de defensivo, contribuindo para diminuição dos impactos ambientais e aumento da segurança no campo (menor risco de intoxicações). Os resultados permitiram identificar vantagens competitivas, com destaque para a praticidade e rapidez, além de permitir controle localizado de plantas daninhas, sem a necessidade de uso de tratores, o qual aumenta compactação do solo e requer maior investimento em equipamentos. A partir destes resultados, serão estabelecidos protocolos de utilização para diversas culturas agrícolas, tais como: Amendoim (Arachis hypogaea L.), Cana-de-açúcar (Saccharum officinarum L.), Soja (Glycine max L.), Milho (Zea mays). 
Palabras clave: culturas; defensivos agrícolas; pragas

\begin{abstract}
The present work had as objective to study and to validate the application of defenses through RPAs (Remotely Piloted Aircraft), commonly denominated Drones. The experiment happened at APTA East Center in Ribeirão Preto, Sao Paulo. The herbicide solution glyphosate $1.5 \mathrm{~kg} \mathrm{ha}^{-1}$ (Roundup WG ${ }^{\circledR}$ ) was used using $15 \mathrm{~L} \mathrm{ha}^{-1}$ of syrup volume. The RPA that overflew the study area at three different altitudes together with three different spray nozzle models in a range of $50 \mathrm{~m} \times 5 \mathrm{~m}$, with water-sensitive papers being distributed in these bands to verify the size, quantity and distribution of drops that fell on the plants. Once the information was counted, it was expected that it would be possible to create different application models, leading to greater efficiency and effectiveness of the applications. In addition, avoid waste of defense, contributing to decrease environmental impacts and increase safety in the field (lower risk of intoxication). The results allowed to identify competitive advantages, highlighting the practicality and speed, besides allowing localized control of weeds, without the need of tractors, which increases soil compaction and requires greater investment in equipment. From these results, use protocols will be established for various agricultural crops, such as: Peanut (Arachis hypogaea L.), Sugarcane (Saccharum officinarum L.), Soya (Glycine max L.), Maize (Zea mays).
\end{abstract}

Keywords: cultures; agricultural defensives; pests

\section{Introdução}

A pulverização agrícola é um método eficaz para controle de pragas e doenças, nas mais variadas áreas de uma cultura. Essa técnica realiza a proteção em escala, permitindo assim, o aumento da produtividade e a segurança da matéria-prima. A pulverização serve para eliminar e controlar plantas invasoras e até mesmo prevenir doenças que possam interferir na qualidade da colheira (Brasquímica, 2017).

Entre as mais diversas técnicas de aplicação de defensivos agrícolas que são encontradas no mercado, as que mais se destacam são as que se baseiam no princípio de fracionamento hidráulico das gotas. Quando se observa somente o modo cujo defensivo agrícola atua sobre o alvo, é errôneo, pois não se observar a técnica de aplicação de defensivos podem acarretar em contaminação do ambiente (Cunha \& Ruas, 2006).

Para os aplicadores, um dos maiores desafios é minimizar essa contaminação ambiental, devido à forte pressão que a sociedade tem colocado, afim de uma produção agrícola socialmente sustentável e ambientalmente segura. As aplicações com essas características são conseguidas, segundo Cunha et al., (2005), com o domínio da técnica de aplicação e a correta escolha da ponta de pulverização (Cunha, et al., 2008).

Atualmente, a tendência é a redução do volume de pulverização, pois adotando esse procedimento, se reduz o número de reabastecimentos, e como consequência, há redução do volume de água necessário para as operações, aumentando então, a capacidade operacional (Boller \& Machry, 2007); (Soares \& Leão, 2008).

Têm-se observado a aplicação de baixos volumes e a redução do diâmetro de gotas, fatores estes que, apresentam maiores capacidades de coberturas atingindo então as diversas partes do alvo com maior facilidade (Cunha, et al., 2006).

Grandes avanços estão acontecendo no setor da pulverização de produtos químicos, notadamente inseticidas, fungicidas e herbicidas. Drones multirotor podem fazer a pulverização em locais com focos de doenças nos cultivos, substituindo, as aplicações em área totais e acarretando em uma 
economia significativa de produtos. Além da redução de produtos, outra vantagem importante a substituição de trabalhores com esses pulverizadores costais, para drones autônomos. Assim, o risco de exposição destes trabalhadores aos agroquímicos, são eliminados, assim, os trabalhadores podem executar outras tarefas, enquanto as lavouras são pulverizadoras pelos Drones.

Os Drones voltados para a pulverização, são equipamentos capazes de operar em áreas de difícil acesso e em locais que aviação agricola nao consegue atuar. Os gastos com insumos se reduzem em até $80 \%$, otimizando recursos e aplicando defensivos no momento e local correto, além dos impactos ambientais serem reduzidos. Esses equipamentos podem atender tanto pequenas culturas quanto grandes, em situações que, pulverização com aviões não é viável economicamente, e tambem em áreas de risco, que podem ser proximas à rede eletrica e árvores (FOCO RURAL, 2018).

As aplicações realizadas de maneira aérea, não se diferem das aplicações com equipamentos terrestres. Segundo Neiva (2007), o uso da tecnologia aérea se apresenta como mais economica e vantajosa, uma vez que reduz o tempo da aplicação e que, o produto pode ser aplicado em condições adversas de solos irrigados ou encharcados, possibiltando assim, maior qualidade e uniformidade de aplicação, que não provoca danos seja de amassar a cultura ou compatacção de solo.

No processo de produção agrícola, a aplicação de defensivos agrícolas é um dos mais exigentes, pois além de atender a área de cultivo, existem os cuidados com a preservação do meio ambiente (Christofoletti, 1999). Enquando que, para Schröder (2005), uma máquina sofisticada será pouco valor, se sua operação não seguir as especificações tecnicas. O segundo autor, descreve os procedimentos operacionais e equipamento adequados, pois estes são os pilares que sustentam as modernas aplicações aérea, com objetivo de impactar de maneira negativa o ambiente. Já é possível utilizar um drone tipo avião para mapear uma grande lavoura e assim, gerar mapas com o posicionamento de focos de plantas daninhas, e assim, pulverizar com herbicidas utilizando o drone multirotor nestes locais em dois ou três dias. A economia de herbicidas chega em até $95 \%$, em relação à pulverização de uma área total (SINDAG, 2018). O objetivo do trabalho foi estudar e validar a aplicação de defensivos agrícolas através de RPAS, identificando eficiencia de operação e entre os modelos de bicos, aquele que é mais eficaz em altura de voo e tamanho de gotas durante a pulverização.

\section{Material e métodos}

O experimento foi realizado na APTA Centro Leste, no município de Ribeirão Preto, São Paulo - Brasil (Figura 1). Foram utilizados: solução de herbicida glifosate $1.5 \mathrm{~kg} \mathrm{ha}^{-1}$ (Roundup WG) com $15 \mathrm{~L} \mathrm{ha}^{-1}$ de volume de calda. O RPA de fabricação Chinesa, distribuído pela Empresa Yuren sobrevoou cada área de interesse, em três diferentes altitudes, sendo elas: $5 \mathrm{~m}, 10 \mathrm{~m}$ e $15 \mathrm{~m}$, juntamente com três diferentes modelos de pontas pulverizadoras, nas cores: amarelo, cinza e verde, estas que, apresentam diferentes vazões (Figura 2).

Em cada uma dessas faixas de aplicação foram distribuídos papeis hidrossensíveis à água, afim de serem quantificados por amostra a quantidade de gotas presentes e também a quantidade total de gotas identificadas em cada faixa do experimento. A contagem e identificação destas, foram realizadas utilizando o software Gotas, desenvolvimento pela Embrapa, que auxilia em trabalhos voltados à pulverização e disponíveis de maneira gratuita aos estudantes.

Depois da contagem, foi verificado de maneira visual os aspectos das gotas. 


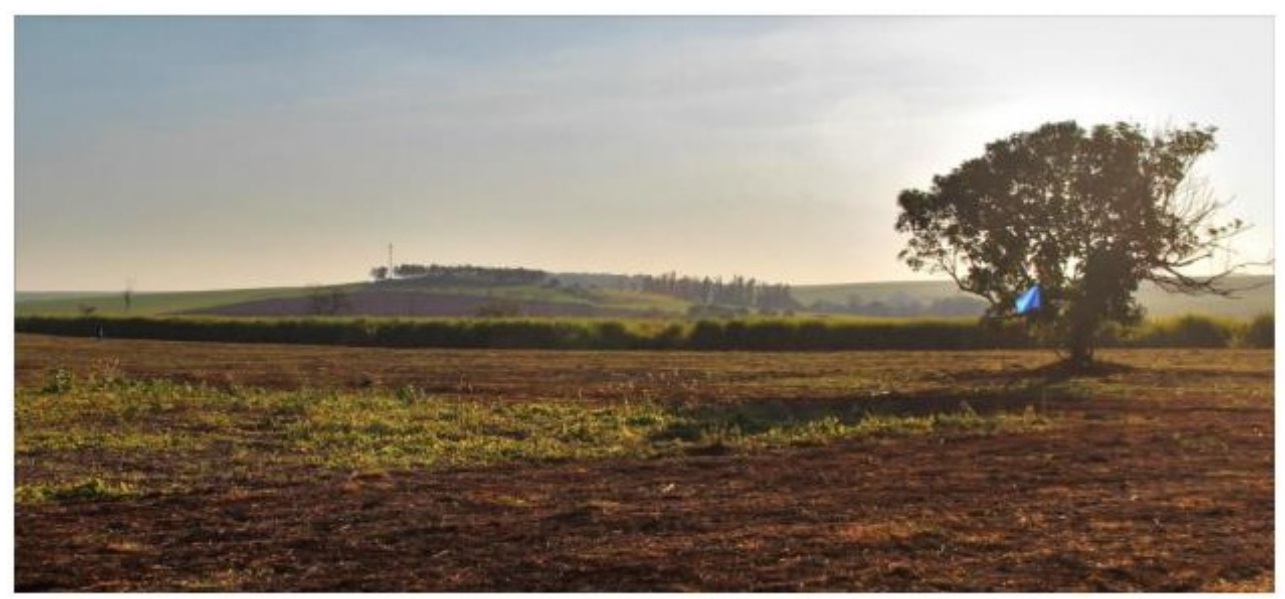

Figura 1. Um dos lados da área demarcada para o experimento.
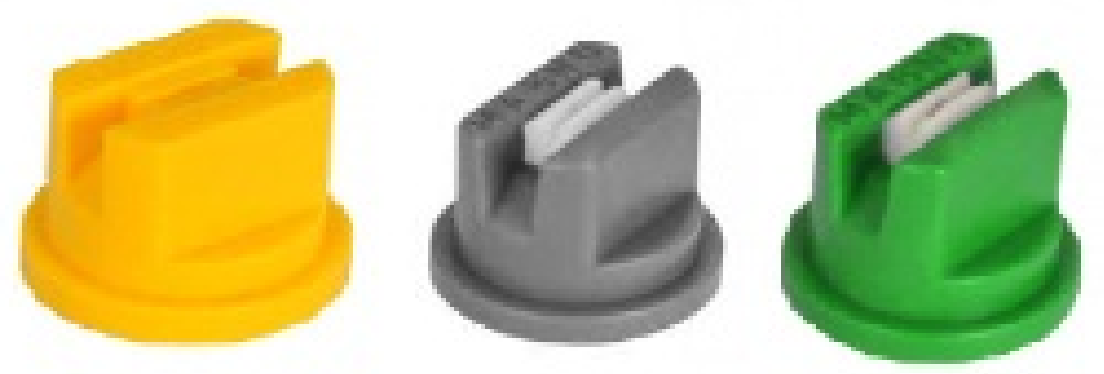

Figura 2. Bicos utilizados para realizar experimento

\section{Resultados e discussão}

A Tabla 1 mostra a quantidade de gotas identificadas, para cada uma das amostras das ponta: amarela, cinza e verde; além das diferentes alturas em que os voos foram realizados.

Tabla 1. Resultados de los parámetros fisicoquímicos del vino de uva Isabella -Huila

\begin{tabular}{cccccccccc}
\hline & \multicolumn{3}{c}{ Ponta Amarela } & \multicolumn{3}{c}{ Ponta Cinza } & \multicolumn{3}{c}{ Ponta Verde } \\
\hline & $5(\mathrm{~m})$ & $10(\mathrm{~m})$ & $15(\mathrm{~m})$ & $5(\mathrm{~m})$ & $10(\mathrm{~m})$ & $15(\mathrm{~m})$ & $5(\mathrm{~m})$ & $10(\mathrm{~m})$ & $15(\mathrm{~m})$ \\
\hline $\mathrm{A} 1$ & 7 & 60 & 77 & 8 & 39 & 86 & 127 & 162 & 72 \\
$\mathrm{~A} 2$ & 9 & 68 & 84 & 62 & 40 & 24 & 152 & 158 & 123 \\
$\mathrm{~A} 3$ & 17 & 97 & 94 & 132 & 58 & 48 & 106 & 197 & 121 \\
$\mathrm{~A} 4$ & 12 & 78 & 123 & 69 & & 23 & 74 & 142 & 144 \\
$\mathrm{~A} 5$ & 7 & 93 & 129 & 73 & & 28 & 89 & 145 & 110 \\
\hline
\end{tabular}

Na Tabla 2, mostra o total de gotas contabilizadas, para cada uma das pontas em suas diferentes alturas de vôo, levando a perceber que, a ponta amarela, em altitudes de 5 e 10 metros, foram contabilizadas, 396 e 507 gotas, sequencialmente. Enquanto que, a ponta cinza, apresentou maior numero de gotas, somente na altitude de 5 metros, 344 gotas. Em todas as altitudes da ponta verde, foram apresentadas mais de 500 gotas, e na altitude de 10 metros, foram contabilizadas 804 gotas. 
Tabla 2. Total de gotas contabilizadas em cada ponta de pulverização.

\begin{tabular}{cccccccccc}
\hline \multicolumn{4}{c}{ TOTAL DE GOTAS CONTABILIZADAS PARA CADA PONTA DE PULVERIZAÇÃO } \\
\hline Cores Pontas & AMARELO & \multicolumn{3}{c}{ CINZA } & \multicolumn{4}{c}{ VERDE } \\
\hline Alturas de voo & $5(\mathrm{~m})$ & $10(\mathrm{~m})$ & $15(\mathrm{~m})$ & $5(\mathrm{~m})$ & $10(\mathrm{~m})$ & $15(\mathrm{~m})$ & $5(\mathrm{~m})$ & $10(\mathrm{~m})$ & $15(\mathrm{~m})$ \\
Total Gotas & 52 & 396 & 507 & 344 & 137 & 209 & 548 & 804 & 570 \\
\hline
\end{tabular}

Quando se observa a quantidade de gotas em em uma altitude de voo de 15 metros apresenta cada cor de ponta, percebe-se que a cor verde, com gotas maiores (Figura 3), se comparada a ponta altitude de 10 metros, foi a mais eficiente durante a verde, com voo realizado em 10 metros (Figura 4). aplicação. Entretanto, quando observamos os papeis hidrossensíveis, percebemos que, a ponta amarela,
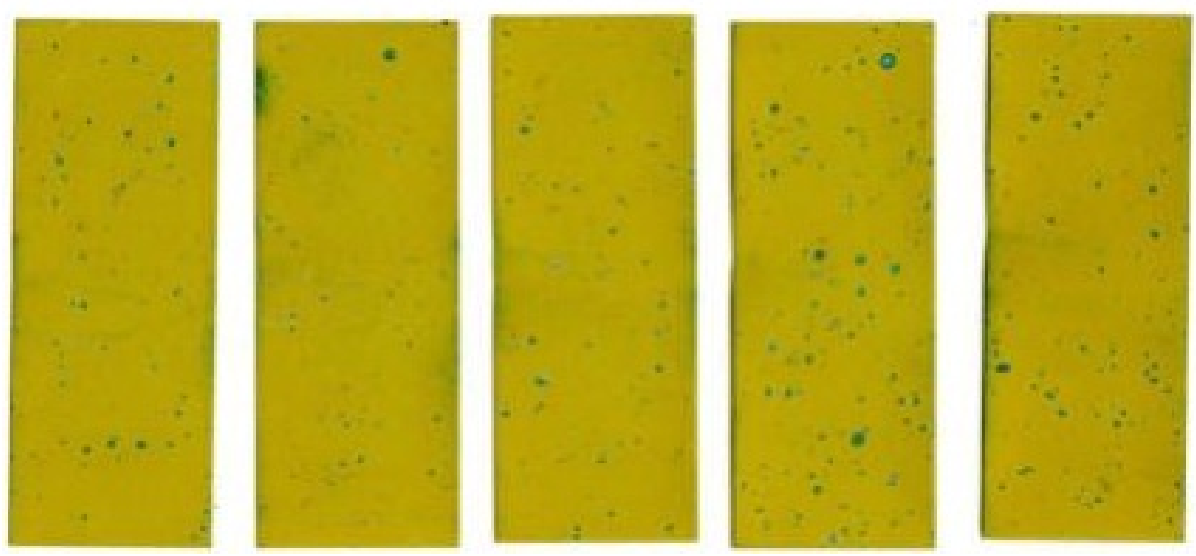

Figura 3. Gotas presentes em papel hidrosenssível, ponta amarela, altitude voo: 15 metros.
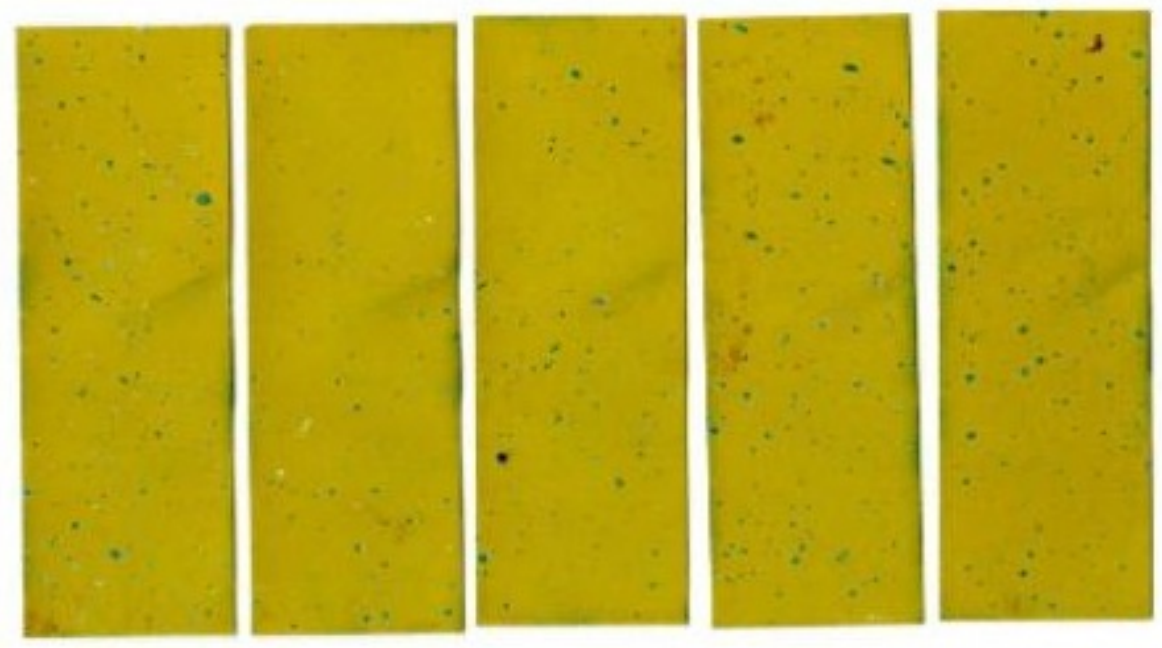

Figura 4. Gotas presentes em papel hidrosenssível, ponta verde, altitude voo: 10 metros.

\section{Conclusões}

É possível identificar oportunidades de aplicações complementares com drones. Os aviões agricolas pulverizam lavouras com rapidez e precisão, mas são limitados, locais com obstáculos, redes elétricas e até mesmo árvores dentro das lavouras. Em pequenas áreas, os drones podem então, complementar a 
aplicação com precisão similiar ou superior ao avião. Paralelamente, os pulverizadores terrestres tratam grandes áreas com precisão, mas alguns pontos da lavoura com solo encharcado, pedregoso ou outros obstáculos podem comprometer a qualidade do serviço. Para evitar que em tais locais as doenças e plantas daninhas se proliferem, os drones fazem aplicações pontuais.

Foi possível concluir que o uso de RPAs para pulverização é viável, pois a partir da existência de plantas invasoras, essas podem ser controladas, e que, voos com 15 metros de altitude, utilizando a ponta amarela acarretam em uma maior área de aplicação por voo e não interfere de forma negativa na eficácia da aplicação.

\section{Referências bibliográficas}

Boller, W., Machry, M., 2007. Efeito da pressão de trabalho e de modelos de pontas de pulverização sobre a eficiência de herbicida de contato em soja. Engenharia Agrícola, Jaboticabal, v.27, n.3, p.722- 727. DOI: https://doi.org/10.1590/s0100-69162007000400015

Brasquímica., 2017. Pulverização Agrícola: O que é e como funciona. Disponível em: http://brasquimica.ind.br/pulverizacao-agricola/ Acesso em 05 ago 2018.

Cunha, J. P. A. R., Moura, E. A. C., Júnior, J. L. S., Zago, F. A., Juliatti, F. C., 2008. Efeito de pontas de pulverização no controle químico da ferrugem da soja. Engenharia Agrícola. Jaboticabal, v.28, n.2, p.283-291. DOI: https://doi.org/10.1590/s0100-69162008000200009

Cunha, J. P. A. R., Reis, E. F., Santos, R. O., 2006. Controle químico da ferrugem asiática da soja em função de ponta de pulverização e de volume de calda. Ciência Rural, Santa Maria, v.36, n.5, p.1360-1366. DOI: https://doi.org/10.1590/s0103-84782006000500003

Cunha, J. P. A. R., Ruas, R. A. A., 2006. Uniformidade de distribuição volumétrica de pontas de pulverização de jato plano duplo com indução de ar. Pesquisa Agropecuária Tropical, Goiânia, v.36, n.1, p.61- 66. DOI: https://doi.org/10.1590/s0100-69162006000200024

Cunha, J. P. A. R., Teixeira, M. M., Vieira, R. F., Fernandes, H. C., 2005. Deposição e deriva de calda fungicida aplicada em feijoeiro, em função de bico de pulverização e de volume de calda. Revista Brasileira de Engenharia Agrícola e Ambiental, Campina Grande, v.9, n.1, p.133-138. DOI: https://doi.org/10.1590/s1415-43662005000100021

Christofoletti, J. C., 1999. Considerações sobre a deriva nas pulverizações agrícolas e seu controle. Teejet South Amercia, São Paulo. 15p.

FOCO RURAL., 2018. Demonstração de Drone de pulverização agrícola. Consultado em 8 de agostos de 2018. http://www.focorural.com/detalh es/n/n/10266/demonstracao-de-drone-de-pulverizac ao-agricola-.html. DOI: https://doi.org/10.11606/t. 3.2013.tde-24112014-153222

Neiva., 2007. Vantagens da Pulverização Aérea. Consultado em 8 de agostos de 2018. http://aeroneiva.com.br/site/content/produtos_ipa nema_vant_pulv.asp

Soares, J., Leão, M., 2008. Optimização da pulverização em médio e baixo volume na produção integrada de pêra rocha. Consultado em 5 de agostos de 2018. http://www.bayercropscience.pt/download/pi_pera _rocha.pdfi. Acesso em: 05 agostos 2018.

Schröder, P. E., 2005. Aviação agrícola em alta rotação: controle de pragas em soja com BVO.

La Revista Ingeniería y Región cuenta con la Licencia Creative Commons Atribución (BY), No Comercial (NC) y Compartir Igual (SA) 